ARTICLE

https://doi.org/10.1038/s41467-019-11965-5

\title{
Giant piezoresistive effect by optoelectronic coupling in a heterojunction
}

Thanh Nguyen"1, Toan Dinh¹, Abu Riduan Md Foisal', Hoang-Phuong Phan', Tuan-Khoa Nguyen', Nam-Trung Nguyen (iD ${ }^{1} \&$ Dzung Viet Dao (i) ${ }^{1,2}$

Enhancing the piezoresistive effect is crucial for improving the sensitivity of mechanical sensors. Herein, we report that the piezoresistive effect in a semiconductor heterojunction can be enormously enhanced via optoelectronic coupling. A lateral photovoltage, which is generated in the top material layer of a heterojunction under non-uniform illumination, can be coupled with an optimally tuned electric current to modulate the magnitude of the piezoresistive effect. We demonstrate a tuneable giant piezoresistive effect in a cubic silicon carbide/silicon heterojunction, resulting in an extraordinarily high gauge factor of approximately 58,000, which is the highest gauge factor reported for semiconductor-based mechanical sensors to date. This gauge factor is approximately 30,000 times greater than that of commercial metal strain gauges and more than 2,000 times greater than that of cubic silicon carbide. The phenomenon discovered can pave the way for the development of ultrasensitive sensor technology.

\footnotetext{
${ }^{1}$ Queensland Micro- and Nanotechnology Centre, Griffith University, Brisbane, Queensland, Australia. ${ }^{2}$ School of Engineering and Built Environment, Griffith University, Gold Coast, Queensland, Australia. Correspondence and requests for materials should be addressed to T.N. (email: thanh.nguyen11@griffithuni.edu.au) or to T.D. (email: toan.dinh@griffith.edu.au) or to D.V.D. (email: d.dao@griffith.edu.au)
} 
$\mathrm{D}$ iscovered by Smith in $1954^{1}$, the piezoresistive effect has been utilized as a major mechanical sensing technology ${ }^{2}$. Piezoresistive sensitivity refers to the fractional change in resistance under applied strain, known as the gauge factor (GF). This sensing technology can be found in a wide range of applications, such as strain, force, pressure, and tactile sensors ${ }^{3}$ and accelerometers ${ }^{4,5}$. The advantages of this sensing concept include, but are not limited to, low power consumption, simple readout circuits and miniaturization capability ${ }^{5}$. However, the performance of the piezoresistive effect depends upon the carrier mobility, which is fundamentally limited by the nature of the piezoresistive materials.

The enhancement of the piezoresistive effect has been of great interest for developing ultra-sensitive sensing devices. The conventional strategy focuses on the arrangement of piezoresistors in optimal crystal orientations $s^{6,7}$. For example, the longitudinal piezoresistive coefficient of p-type silicon (100) is $\sim 6.6 \times 10^{-11} \mathrm{~Pa}^{-1}$ in the [100] direction, while its value increases to $71.8 \times 10^{-11} \mathrm{~Pa}^{-1}$ in the [110] direction ${ }^{1,6}$. The GF of single crystalline p-type cubic silicon carbide (3C-SiC) is 5.0 and 30.3 in the [100] and [110] orientation, respectively ${ }^{8}$. A significant improvement in piezoresistive sensitivity has also been demonstrated using optimaldoping concentrations ${ }^{6,9}$. In terms of material choice, metal strain gauges have been commercialized and are widely employed in industry, research and daily life. However, the piezoresistive effect in metals is fundamentally based on a geometry change under applied strain, resulting in a low GF that is typically less than $2^{2}$. Semiconductors such as silicon $(\mathrm{Si})$ and silicon carbide $(\mathrm{SiC})$ have emerged as suitable materials for strain sensing because of their relatively high $\mathrm{GF}$ of up to $200 \mathrm{in} \mathrm{Si}^{2}$ and $30 \mathrm{in} \mathrm{SiC}^{8,10}$. While the strain-induced geometry change can be neglected in these semiconductors, the carrier mobility governs the piezoresistive performance.

Interestingly, a significant enhancement of the piezoresistive effect can be achieved by scaling down piezoresistors to the nanometer-scale owing to advanced nanofabrication techniques. At the nanoscale level, the charge mobility and surface-to-volume ratio considerably increase, resulting in a significant improvement in the strain sensitivity ${ }^{11-13}$. For instance, a large piezoresistive effect in top-down fabricated silicon nanowires (SiNWs) has been observed with a longitudinal piezoresistive coefficient of up to $-3550 \times 10^{-11} \mathrm{~Pa}^{-1}$, which is almost 38 times greater than that of bulk $\mathrm{Si}^{13}$. However, the reliability of a large piezoresistive effect on the nanoscale is still controversial ${ }^{14-17}$.

More recently, coupling piezoresistivity with other physical effects, such as piezoelectricity, has emerged as an advanced and promising approach to boost piezoresistivity. As such, the strain-modulated electric potential in piezoelectric materials, known as piezotronics, can be used to control or tune the transport of charge carriers. By utilizing strain-induced piezoelectric polarization charges at the local junction of zinc oxide $(\mathrm{ZnO})$ nanowires to modify their energy band structures, Jun Zhou et al. ${ }^{18}$ successfully demonstrated an increase in the GF from 300 to 1250 , when the strain increased from $0.2 \%$ to $1 \%$. Additionally, an electrically controlled giant piezoresistive effect in SiNWs has been reported with a GF of up to 5000 by employing an electrical bias to manipulate the charge carrier concentration $^{19}$. Coupling of multiple physical effects in nanostructures has also been employed to modulate electrical transport in logic circuits $^{20}$, enhance the sensitivity and detection resolution of bio/chemical sensors ${ }^{21-23}$, and improve the photovoltaic performance of solar cells ${ }^{24}$. An enhancement of up to $76 \%$ of the output voltage has been revealed in solar cells by modulating the interfacial charge transfer in indium phosphide/zinc oxide ( $\mathrm{InP} / \mathrm{ZnO}$ ) heterojunctions by applying temperature gradients across the device ${ }^{24}$.
In the present work, we report the discovery of a giant piezoresistive effect in semiconductor heterojunctions by coupling the photoexcitation of the charge carriers, the strain modification of the carrier mobility and the electric field modulation of the carrier energy. Visible light is utilized to illuminate the top layer material of the heterojunction structure, in which the sensing element is non-uniformly illuminated by a vertical visible light. This illumination generates a lateral photovoltage that is counteracted by an externally controlled electric field to tremendously modulate the magnitude of the piezoresistive effect. As a proof of principle, we employ a $3 \mathrm{C}-\mathrm{SiC}$ nanofilm grown on a Si substrate to form a $3 \mathrm{C}-\mathrm{SiC} / \mathrm{Si}$ heterojunction. Under visible light illumination, a stable GF value of the $\mathrm{SiC} / \mathrm{Si}$ heterojunction as high as $\sim 58,000$ is achieved, which is the highest value ever reported for semiconductor piezoresistive sensors. The piezoresistive effect in the $\mathrm{SiC}$ nanofilm is utilized to detect mechanical stress or strain, while its sensitivity is boosted by optimally and simultaneously regulating both the lateral photovoltage and the tuning current. While heavily doped p-type $3 \mathrm{C}-\mathrm{SiC} / \mathrm{p}$-type $\mathrm{Si}\left(\mathrm{p}^{+}-3 \mathrm{C}-\mathrm{SiC} / \mathrm{p}-\mathrm{Si}\right)$ is used in this work, our method could also be extended to enhance the sensitivity of other materials and smart structures that have simultaneous photovoltaic and piezoresistive properties. Thus, our findings can open a new era for the development of ultrasensitive mechanical sensors.

\section{Results}

Enhancement of the piezoresistive effect. We demonstrated an unprecedentedly large piezoresistive effect in $\mathrm{p}^{+}-3 \mathrm{C}-\mathrm{SiC} / \mathrm{p}-\mathrm{Si}$ heterojunctions using a bending method. The carrier concentrations in the $3 \mathrm{C}-\mathrm{SiC}$ nanofilm and $\mathrm{Si}$ substrate were $5 \times 10^{18}$ and $5 \times 10^{14} \mathrm{~cm}^{-3}$, respectively. The light intensity was $19,000 \mathrm{~lx}$, while three different strains of 225,451 , and $677 \mathrm{ppm}$ were induced in the material (Fig. 1). In our experiments, we supplied an optimally controlled tuning current and simultaneously measured the output voltage.

We supplied a constant tuning current of $29.75 \mu \mathrm{A}$ and light with an intensity of 19,000 lx. Strain was periodically applied (i.e., Load ON) and released (i.e., Load OFF). The fractional change in

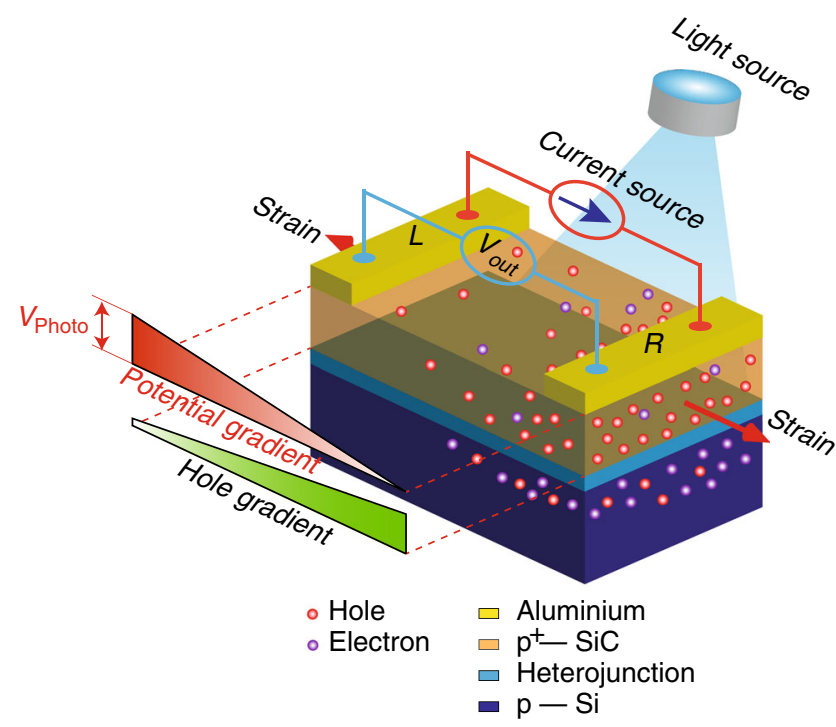

Fig. 1 The sensitivity enhancement by optoelectronic coupling. The piezoresistive effect in the cubic silicon carbide/silicon heterojunction was modulated by non-uniform visible light illumination on the surface of the sensing element coupled with an optimally controlled tuning current 
a

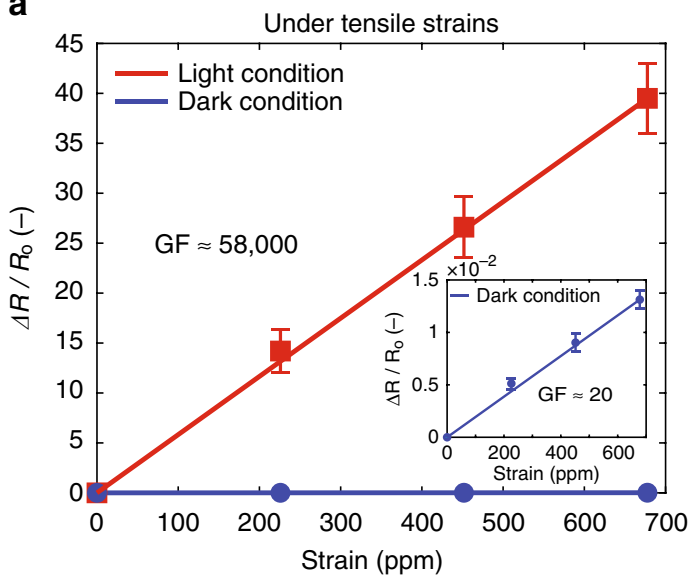

C

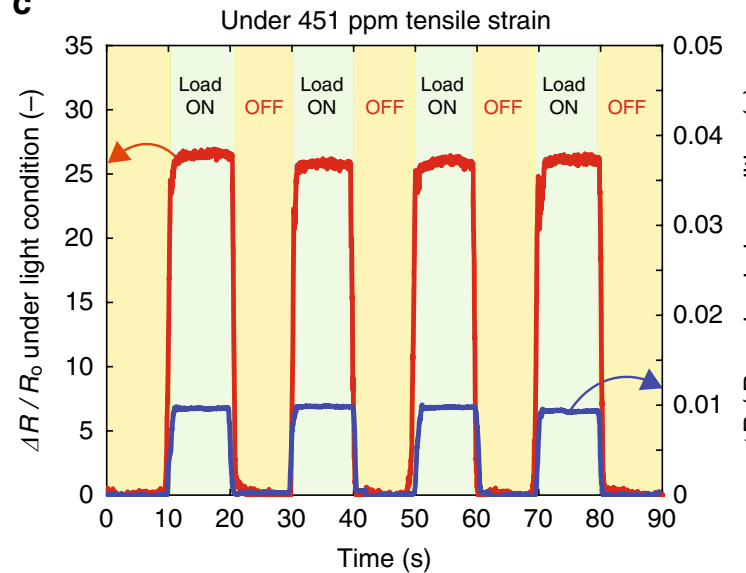

b

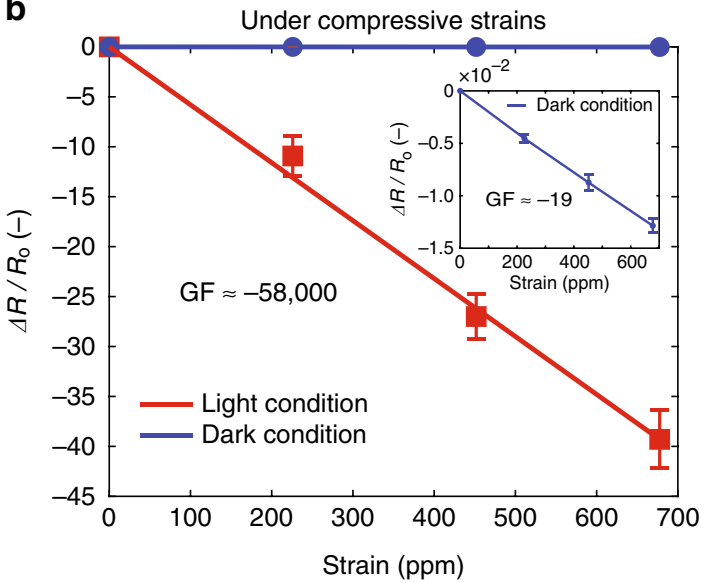

d

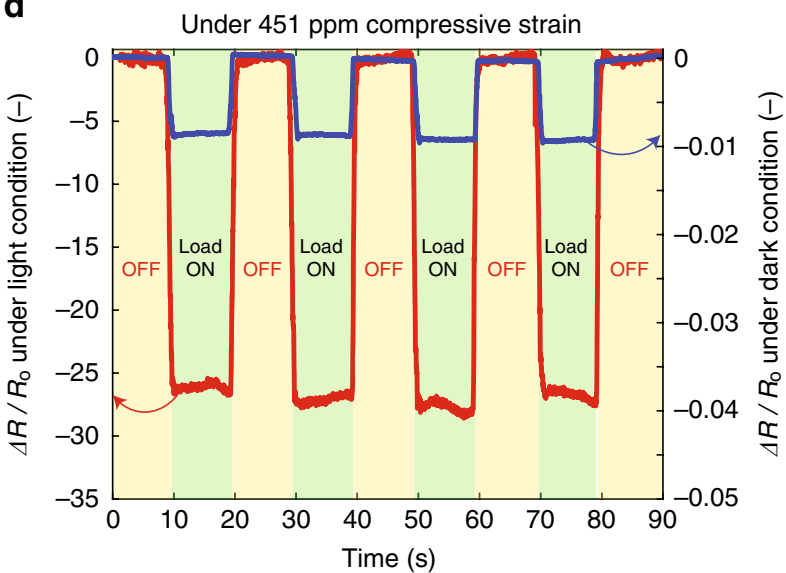

Fig. 2 Enhancement of the piezoresistive effect. An unprecedented high gauge factor (GF) was achieved by simultaneously utilizing the lateral photovoltage and tuning the electric current to modulate the performance of the piezoresistive effect under tensile strain $\mathbf{a}$ and compressive strain $\mathbf{b}$. The linearity of the sample applied in this method is excellent. Due to our discovery, a GF as high as 58,000 was achieved under tensile and compressive strain. c Repeatability of the fractional change in resistance under dark and light conditions as 451 ppm tensile strain was periodically turned ON and OFF. d Repeatability of the fractional change in resistance under dark and light conditions as the $451 \mathrm{ppm}$ compressive strain was periodically turned ON and OFF

the resistance $\left(\Delta R / R_{0}\right)$ was calculated as follows:

$$
\frac{\Delta R}{R_{0}}=\frac{R-R_{0}}{R_{0}}=\frac{\frac{V}{I}-\frac{V_{0}}{I}}{\frac{V_{0}}{I}}=\frac{V-V_{0}}{V_{0}}=\frac{\Delta V}{V_{0}}
$$

where the strain-free resistance $R_{0}$ is calculated by $R_{0}=V_{0} / I, V_{0}$ is the voltage measured between the two electrodes under strainfree conditions, and $I$ is the supplied tuning current flowing between the two electrodes, which was kept constant throughout the measurement. When a strain or stress is applied, the resistance $R$ will change due to the piezoresistive effect. The value of resistance is calculated by $R=V / I$, where $V$ is the voltage measured between two electrodes under stress/strain application. As shown in Fig. 2a, b, the fractional changes in the resistance $\Delta R / R_{0}$ linearly increased with the increases in the tensile and compressive strain, which is desirable for high-performance strain-sensing applications. Figure $2 c$ compares the fractional changes in the resistance $\Delta R / R_{0}$ between dark and light conditions under $451 \mathrm{ppm}$ tensile strain, while Fig. $2 \mathrm{~d}$ shows $\Delta R / R_{0}$ under $451 \mathrm{ppm}$ compressive strain. Under a $451 \mathrm{ppm}$ tensile strain, the $\Delta R / R_{0}$ value increased $\sim 2950$ times from 0.009 in the dark condition to 26.6 under light illumination, and this trend was similar under the compressive strain (the $\Delta R / R_{0}$ value increased from -0.0087 to -27 under the $451 \mathrm{ppm}$ compressive strain). These results indicate a giant enhancement in the piezoresistive effect under light conditions. This tremendous enhancement was confirmed under other applied strains as well (Supplementary Fig. 1). The piezoresistive sensitivity is characterized by the GF, which is defined as the fractional resistance change in response to the applied strain:

$$
\mathrm{GF}=\frac{\Delta R}{R_{0}} \times \frac{1}{\epsilon}=\frac{\Delta V}{V_{0}} \times \frac{1}{\epsilon}
$$

where $\varepsilon$ is the applied strain, which is detailed in Supplementary Table 1. Under the tensile strain, the GF was found to be 20 in the absence of light (the inset in Fig. 2a) and increased to $\sim 58,000$ under light illumination (Fig. 2a), which is the highest strain sensitivity ever reported. Moreover, under compressive strain, the change in the resistance or GF was similar to that observed under tensile strain but opposite in sign (Fig. 2b). In addition, the signal to noise ratio (SNR) under light condition increased significantly in comparison with that under dark condition.

Tuning current in optoelectronic coupling. The dependence of the piezoresistive effect on the tuning current under the $451 \mathrm{ppm}$ tensile strain and the illumination condition of 19,000 lx intensity is depicted in Fig. 3. Figure 3a shows the change in the GF as the tuning current increased from 15 to $45 \mu \mathrm{A}$, while Fig. 3b-d illustrate the magnified graphs of the GF value versus the tuning current in three distinguished current ranges. The GF increased from approximately -16 to -1800 as the current increased from 


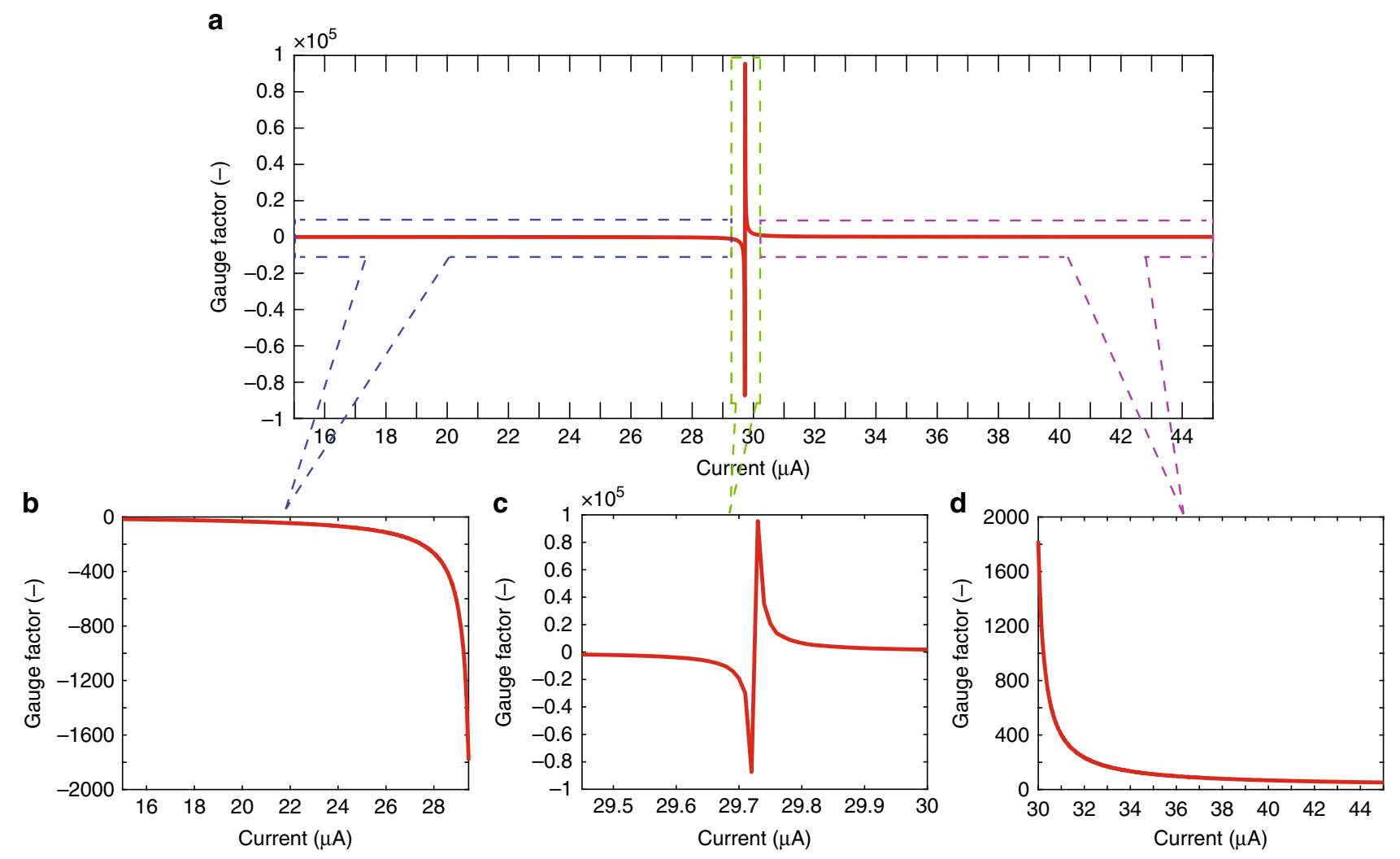

Fig. 3 The role of the tuning current in the enhancement of the piezoresistive effect. a Characteristics of GF with respect to the supplied tuning current. Three ranges of tuning current are magnified in $\mathbf{b}$, the negative GF range; in $\mathbf{c}$, the optimal range; and in $\mathbf{d}$, the positive GF range. Under the same light condition, the sensitivity (i.e., GF) changed significantly versus the supplied current. Under a light intensity of 19,000 Ix, as the supplied current was swept from 15 to $45 \mu \mathrm{A}$, the GF increased from approximately -16 to a maximum of $\sim 95,500$, and then decreased to about $\sim 50$

15 to $29.45 \mu \mathrm{A}$ (Fig. $3 \mathrm{~b}$ ), while it decreased from 1800 to $\sim 50$ for the high current ranging from 30 to $45 \mu \mathrm{A}$ (Fig. 3d). This difference was attributed to the dominance of the photomodulated potential over the injected potential (i.e., tuning current). The compensation of these two potentials in the current range of from 29.45 to $30 \mu \mathrm{A}$ led to a change in the GF sign and the ultra-high absolute GF values (Fig. 3c). As the strain-free voltage $V_{0}$ was relatively small due to the potential compensation, the modulation of the charge mobility under strain resulted in a significant change in the measured voltage, resulting in an ultra-high GF. It was observed that a higher GF can be achieved as the magnitude of the tuning current became closer to that of the photocurrent. For instance, under an incident light intensity of 19,000 lx, the maximum GF observed was as high as $\sim 95,500$. However, as the tuning current became closer to the photocurrent, the GF was more variable due to an inevitable slight variation in the photocurrent. Therefore, to achieve a higher stable sensitivity, the tuning current should be controlled and maintained as close as possible to the magnitude of the photocurrent but also far enough to retain stability. In our experiment, we achieved a stable GF of $\sim 58,000$ when the tuning current was help constant at $29.75 \mu \mathrm{A}$ under an illumination of $19,000 \mathrm{~lx}$ from a stable visible light source.

As such, the significant enhancement in the piezoresistive effect by optoelectronic coupling in 3C-SiC/Si heterojunctions is a combination of two key elements: light illumination and tuning current. This enhancement was first attributed to the photogenerated electrical potential in the $3 \mathrm{C}-\mathrm{SiC}$ film with nonuniform illumination of visible light, which was indicated by the lateral photovoltage and/or photocurrent. The magnitudes of the photovoltage and the photocurrent can be manipulated by parameters, such as light intensity, light position, or light wavelength. The lateral photovoltage, for example, was measured to be approximately $-9 \mathrm{mV}$ under a light intensity of $19,000 \mathrm{~lx}$ (Fig. 4a), and the value of the photocurrent was $\sim 29.7 \mu \mathrm{A}$ (Fig. 4b). The magnitudes of the generated photovoltage and photocurrent can be changed by changing the light position. For instance, using the same previous light, we gradually adjusted the light beam position from the left (L) electrode to the right $(\mathrm{R})$ electrode (Supplementary Fig. 2). The measured voltage decreased from a large positive value (e.g., $9 \mathrm{mV}$ ) at electrode $\mathrm{L}$ to zero at the center of the device and then increased to a large negative value at electrode $\mathrm{R}$ (e.g., $-9 \mathrm{mV}$ ). The underlying physics behind the generation of the photocurrent and photovoltage on $3 \mathrm{C}-\mathrm{SiC}$ can be explained according to the lateral photoeffect $^{25}$. Figure $5 \mathrm{a}$ shows the photon excitation of electron-hole pairs (EHPs) in the 3C-SiC/Si platform under light illumination. As such, photogenerated charge carriers have a high concentration at electrode $\mathrm{R}$ close to the light source. The formation of the gradient of charge carriers is discussed as follows.

Formation of the gradient of charge carriers. When heavily doped p-type 3C-SiC and p-type $\mathrm{Si}$ are brought together, holes diffuse from the $3 \mathrm{C}$-SiC film into the $\mathrm{Si}$ substrate to decrease the hole gradient, leaving behind negative charges in the SiC layer near the interface of the heterojunction. In contrast, electrons in $\mathrm{Si}$, as minor carriers, migrate into $\mathrm{SiC}$ and create a positive charge layer on the Si side. The migration of electrons and holes forms a depletion region (space charge region) and a built-in electric field $E_{0}$, which bends the conduction band and valence band in the 
a

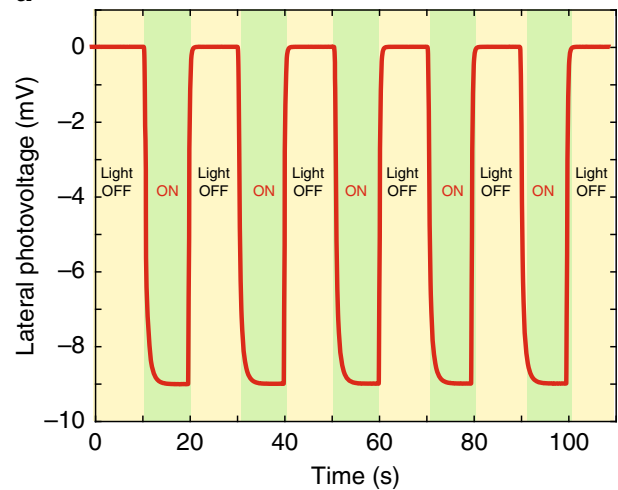

b

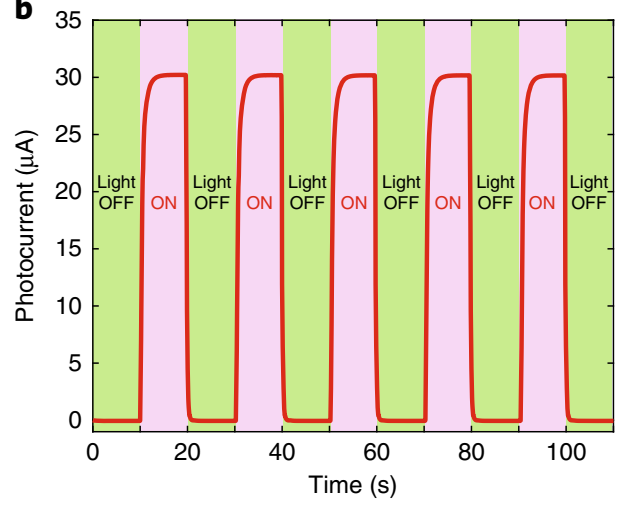

Fig. 4 Generated photovoltage and photocurrent. a Under light illumination of 19,000 Ix, a lateral photovoltage of approximately $-9.0 \mathrm{mV}$ was generated between the two electrodes. $\mathbf{b}$ The photocurrent under light illumination of 19,000 Ix. As the light was turned OFF, the photocurrent was $0 \mu \mathrm{A}$, and this value was $\sim 29.7 \mu \mathrm{A}$ when light with an intensity of $19,000 \mathrm{~lx}$ was ON

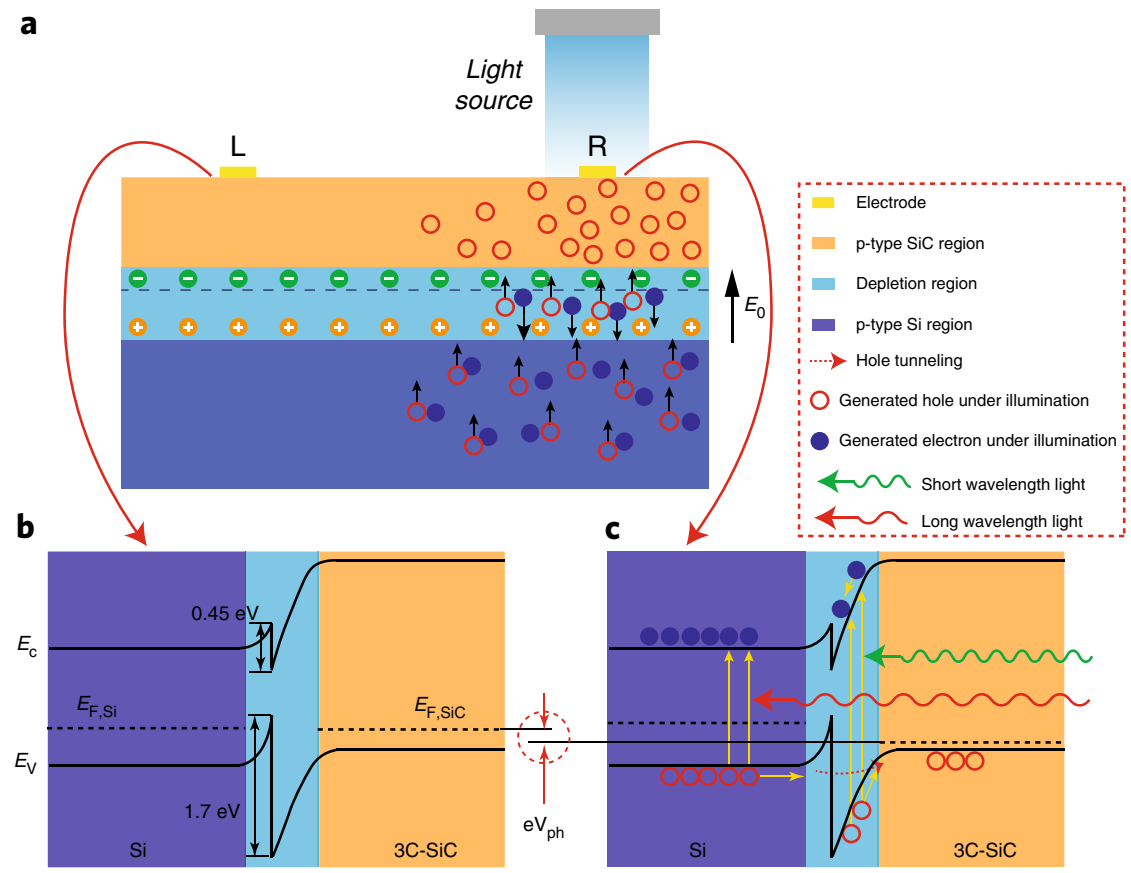

Fig. 5 Formation mechanism of the lateral photovoltage. a Electron-hole pair generation and separation. Under visible light, electron-hole pairs are generated in the depletion region and Si substrate. Then, the generated EHPs in the depletion region are separated by an internal electric field, and the generated holes in the Si substrate tunnel to the 3C-SiC thin film. Schematic band diagrams of the heterojunction at the electrode $\mathrm{L}$ area without illumination $\mathbf{b}$ and at the electrode $\mathrm{R}$ area with illumination $\mathbf{c}$. Under non-uniform illumination, photons are injected into the electrode $\mathrm{R}$ area rather than into the electrode $\mathrm{L}$ area, resulting in holes generated and injected in this area. Consequently, there is a gradient of the hole concentration in the $3 \mathrm{C}$ $\mathrm{SiC}$ layer

depletion region. It is worth noting that the depletion region extends primarily into the Si substrate (Fig. 5a) because the carrier concentration in the $\mathrm{Si}$ substrate $\left(5 \times 10^{14} \mathrm{~cm}^{-3}\right)$ is much lower than that in the $\mathrm{SiC}$ thin film $\left(5 \times 10^{18} \mathrm{~cm}^{-3}\right)$. As shown in Fig. 5b, c, there are energy offsets of 0.45 and $1.7 \mathrm{eV}$ for the conduction band and valence band, respectively, between $3 \mathrm{C}-\mathrm{SiC}$ and $\mathrm{Si}^{26}$. Figure $5 \mathrm{~b}, \mathrm{c}$ present the band energy diagrams of $\mathrm{SiC} / \mathrm{Si}$ under light illumination at electrode $\mathrm{L}$ and $\mathrm{R}$ areas, respectively. Owing to the visible-blind property of $\mathrm{SiC}$, photons were only absorbed in the depletion region and the Si layer, where EHPs were generated. The generated EHPs in the depletion region were separated by the internal electric field $E_{0}$. Consequently, photogenerated holes in the depletion region moved to the $\mathrm{SiC}$ film and increased its electrical conductivity. We hypothesized that the photogenerated holes in $\mathrm{Si}$ also moved towards $\mathrm{SiC}$ by the tunneling mechanism. Under non-uniform illumination, the majority of photons migrated into the electrode $\mathrm{R}$ area rather than into the electrode $\mathrm{L}$ area, resulting in holes were injected into this area. Consequently, there was a potential gradient in the hole concentration from electrode $\mathrm{R}$ to electrode $\mathrm{L}$, which resulted in a difference in the electric potential described as $e V_{\mathrm{ph}}=E_{\mathrm{F}, \mathrm{SiC} @ \mathrm{R}}-$ $E_{\mathrm{F}, \mathrm{SiC} @ \mathrm{~L}}$, where $e$ is the elementary charge, $E_{\mathrm{F}, \mathrm{SiC}}$ is the Fermi energy level, and $V_{\mathrm{ph}}$ is the generated lateral photovoltage. When the external circuit was shorted, the only current in the circuit was the photocurrent $\left(I_{\text {photo }}\right)$.

Hole redistribution. The potential gradient of the hole concentration from $\mathrm{R}$ to $\mathrm{L}$ can also be represented in the $E-k$ (energy-momentum) diagrams in Fig. 6. Under inhomogeneous illumination of light (Fig. 6a), the difference in the 

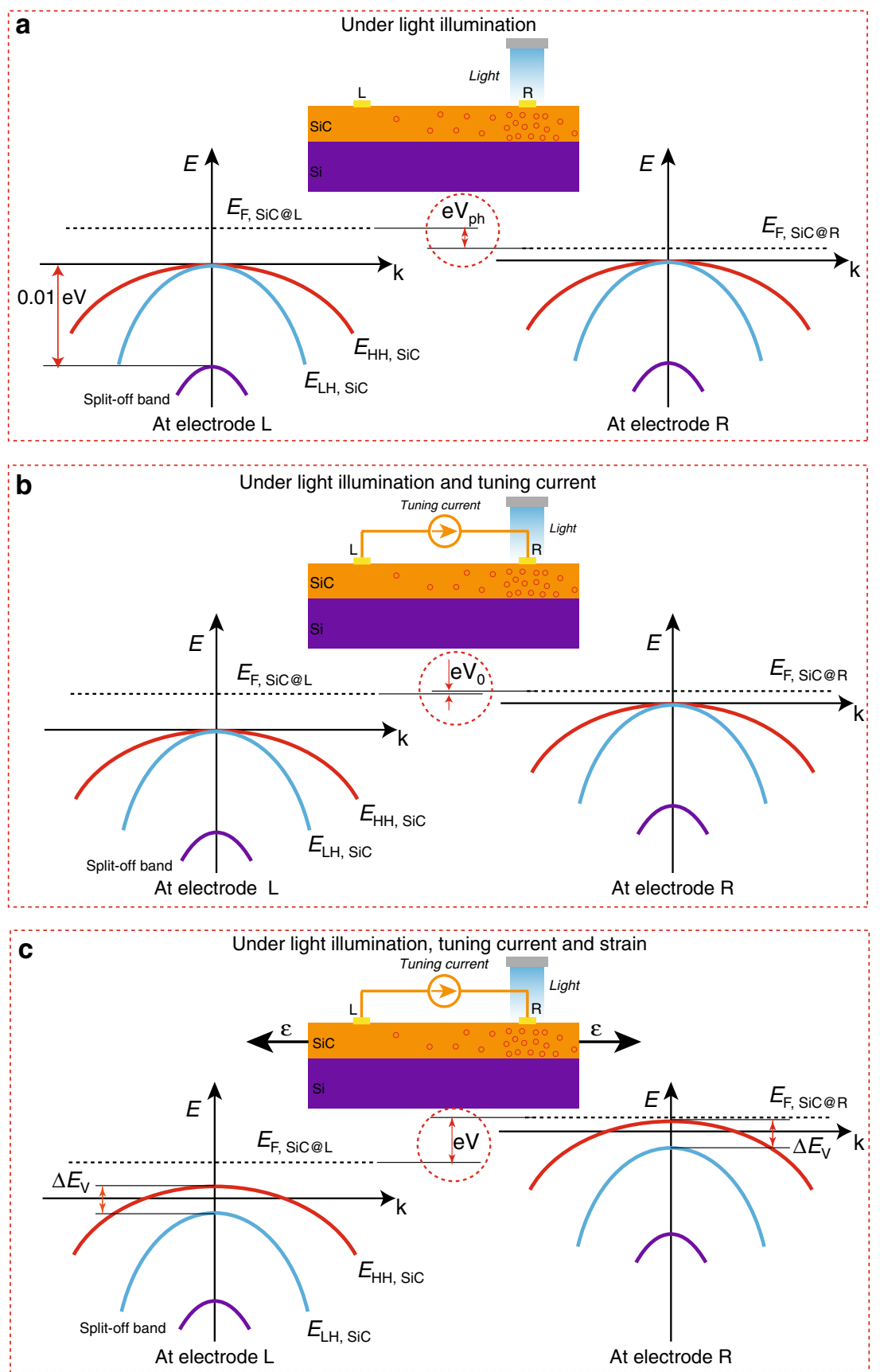

Fig. $6 E-k$ (energy-momentum) characteristics of $3 \mathrm{C}-\mathrm{SiC}$ nanofilms under different conditions. a $E-k$ characteristics at electrode $\mathrm{L}$ and electrode $\mathrm{R}$ under illumination. b Under illumination and tuning current. c Under illumination, tuning current and applied strain

photogenerated hole concentration at $\mathrm{R}$ and $\mathrm{L}$ resulted in a difference in the Fermi levels in the SiC thin film in the two electrode regions. When a bias current $j$ with a positive terminal at electrode $\mathrm{R}$ and a negative terminal at electrode $\mathrm{L}$ is applied, the $3 \mathrm{C}$-SiC band energy was bent upwards from electrode $\mathrm{L}$ to electrode R (Fig. 6b). This bias current created an electric field $E_{\mathrm{b}}$

$$
E_{\mathrm{b}}=\int_{\mathrm{L}}^{\mathrm{R}} j \cdot \frac{1}{\sigma} \mathrm{d} x
$$

where $\sigma$ and $x$ are the conductivity of $\mathrm{SiC}$ and the distance from electrode $\mathrm{L}$, respectively. This electric field $E_{\mathrm{b}}$ offset the lateral photogenerated electric field $E_{\mathrm{ph}}=e V_{\mathrm{ph}}$, resulting in a relatively small voltage $V_{0}$ between the two electrodes. Particularly, under a light intensity of $19,000 \mathrm{~lx}$, a bias current of $29.75 \mu \mathrm{A}$ almost canceled out the lateral photovoltage, resulting in a nearly zero voltage $\left(V_{0} \approx 0\right)$ (Fig. $6 \mathrm{~b}$ ). Figure $6 \mathrm{c}$ shows the change in the band diagram at the electrodes under uniaxial tensile strain. The energy sub-band of heavy holes (HHs) was shifted up to a lower energy level, while the energy sub-band of light holes (LHs) moved down to a higher energy level 27,28 . As a consequence, there was an increase in the $\mathrm{HH}$ concentration and a decrease in the $\mathrm{LH}$ concentration, while the total concentration of holes was hypothesized to be unchanged due to the high doping concentration. It should be noted that as $\mathrm{HHs}$ have a higher effective mass than LHs, the increase in the $\mathrm{HH}$ concentration and the decrease in the LH concentration caused an increase in the total of the effective mass. Consequently, the mobility of the holes was reduced, which diminished the conductivity $\sigma$ or increased resistance. As a result, the bias current generated a high electric field $E$ and a high measured voltage $V$. The significant difference between the 
voltage $V$ under light illumination coupled with the applied strain (Fig. 6c) with respect to the nearly zero voltage $V_{0}$ under the strain-free state (Fig. 6b) led to the giant piezoresistive effect in the $\mathrm{SiC}$ nanofilms. Furthermore, in principle, it is possible to tune $V_{0}$ towards zero by regulating the illumination conditions and tuning the current to achieve a desirable high sensitivity to strain.

\section{Discussion}

We discovered a giant GF of 58,000 in 3C-SiC/Si heterojunctions under optoelectronic coupling. This is the highest GF reported to date, which is $\sim 30,000$ times greater than the GF of commercial metal strain gauges and more than 2000 times higher than that of 3C-SiC under dark conditions. We analyzed three key parameters that contributed to this tuneable giant piezoresistive effect. First, non-uniform illumination created a gradient of carrier concentration within the $3 \mathrm{C}-\mathrm{SiC}$ nanofilm, generating a lateral photovoltage in this layer. Second, the tuning current was introduced to reduce the difference in the Fermi energy levels of $3 \mathrm{C}-\mathrm{SiC}$ at the two electrodes ( $\mathrm{L}$ and $\mathrm{R}$ ). Depending on the value of the lateral photovoltage, the optimal tuning current can have different values. Third, mechanical stress or strain caused shifts in the valance subbands (light hole and heavy hole), leading to the redistribution of charge carriers among these bands and changing the mobility and electrical conductivity of the material. The discovery of this optoelectronic coupling phenomenon in semiconductor heterojunctions has great potential to open a new era of ultra-sensitive sensor technology.

\section{Methods}

Growth of 3C-SiC on a Si substrate. Single crystalline cubic silicon carbide (3C$\mathrm{SiC}$ ) was grown on a single crystalline $\mathrm{Si}$ substrate by low pressure chemical vapor deposition in a $1000{ }^{\circ} \mathrm{C}$ reactor. Ultra-pure silane and acetylene were used as precursor materials to provide $\mathrm{Si}$ and $\mathrm{C}$ in the $3 \mathrm{C}-\mathrm{SiC}$ growth process. Heavily doped $3 \mathrm{C}$-SiC was then formed by doping aluminum atoms from the $\left(\mathrm{CH}_{3}\right)_{3} \mathrm{Al}$ (trimethylaluminium) precursor compound in the in situ growth process. The characteristics of single crystalline $3 \mathrm{C}-\mathrm{SiC}$ on a single crystalline $\mathrm{Si}$ substrate are shown in Supplementary Fig. $3^{14}$. Supplementary Figure $3 a$ (the selected area electron diffraction image) and $3 \mathrm{~b}$ (X-ray diffraction analysis) indicate that single crystalline $3 \mathrm{C}-\mathrm{SiC}$ was epitaxially grown on a single crystalline $\mathrm{Si}$ substrate. The transmission electron microscopy image in Supplementary Fig. 3c confirms the crystalline properties of the $\mathrm{SiC}$ film. The thickness of the $3 \mathrm{C}-\mathrm{SiC}$ layer measured by NANOMETRICS Nano-Spec-based measurements was $300 \mathrm{~nm}$ with the tolerance across the wafer within $\pm 2 \mathrm{~nm}$. The carrier concentrations in the $3 \mathrm{C}$-SiC layer and single crystalline Si substrate were $5 \times 10^{18}$ and $5 \times 10^{14} \mathrm{~cm}^{-3}$, respectively, as determined by the hot probe and Hall effect techniques.

\section{Sample fabrication. To demonstrate the piezoresistive effect of optoelectronic coupling in heterojunctions, cantilevers were fabricated according to the design shown in Supplementary Fig. 4. The length, width, and thickness of the cantilevers were 32,5 , and $0.63 \mathrm{~mm}$, respectively. The distance from the free end of the cantilever to the centre of the piezoresistor was $25 \mathrm{~mm}$. The dimensions of the piezoresistor were $0.5 \mathrm{~mm} \times 2.5 \mathrm{~mm}$, while those of the electrodes were $0.8 \mathrm{~mm} \times$ $2.5 \mathrm{~mm}$. Five cantilevers were fabricated following the process presented in Sup- plementary Fig. 5. After the growth process, an aluminum layer was deposited on top of $3 \mathrm{C}-\mathrm{SiC}$ by a sputtering process. Then, a photoresist layer was coated on the surface of aluminum by a spin-coating technique at a spinning speed of $3500 \mathrm{rpm}$, and the photoresist layer was baked at $110^{\circ} \mathrm{C}$ for $100 \mathrm{~s}$. Next, the wafer was exposed to ultraviolet light to pattern the shape of the electrodes. The aluminum electrodes were formed through an aluminum wet etching process, which was followed by a final dicing process to form the cantilevers. A root mean square roughness of the top surface of the cantilever estimated by atomic force microscopy measurements was smaller than $15 \mathrm{~nm}$. As shown in Supplementary Fig. 6, the $I-V$ characteristics were linear under both dark and light conditions, which confirmed that Ohmic contact formed between aluminum and $3 \mathrm{C}-\mathrm{SiC}$.}

Optoelectronic coupling characterization. To characterize the optoelectronic coupling effect, five cantilevers were tested under the same conditions with the same procedure. As shown in Supplementary Fig. 7, the cantilevers were mounted on the chuck of the EP 6 CascadeMicrotech probe system. The sample position could be accurately adjusted. The samples were illuminated by vertical visible light from the fibre-optic illuminator used in the EP 6 CascadeMicrotech probe system, and the light beam position could also be precisely controlled using a precision XYZ stage. The light intensity measured using a digital lux meter was 19,000 lx.
The tensile and compressive strains on the $\mathrm{SiC}$ devices were induced using a cantilever bending experiment. Three different weights of 50,100, and $150 \mathrm{~g}$ were hung on the free end of the cantilever to induce strain in the sensing element. The strain calculation is detailed in Supplementary Note 1. We controlled the tuning electric current and simultaneously measured the voltage between the two electrodes using a Keithley 2450 SourceMeter.

\section{Data availability}

All relevant data of this work is available from the corresponding author upon reasonable request.

Received: 4 February 2019 Accepted: 2 August 2019

Published online: 12 September 2019

\section{References}

1. Smith, C. S. Piezoresistance effect in germanium and silicon. Phys. Rev. 94, 42 (1954).

2. Barlian, A. A., Park, W.-T., Mallon, J. R., Rastegar, A. J. \& Pruitt, B. L. Semiconductor piezoresistance for microsystems. Proc. IEEE 97, 513-552 (2009).

3. Ho, V. A., Dao, D. V., Sugiyama, S. \& Hirai, S. Development and analysis of a sliding tactile soft fingertip embedded with a microforce/moment sensor. IEEE Trans. Robot. 27, 411-424 (2011).

4. Dao, D. V., Nakamura, K., Bui, T. T. \& Sugiyama, S. Micro/nano-mechanical sensors and actuators based on SOI-MEMS technology. Adv. Nat. Sci.: Nanosci. Nanotechnol. 1, 013001 (2010).

5. Phan, H.-P. et al. Piezoresistive effect in p-type $3 \mathrm{C}-\mathrm{SiC}$ at high temperatures characterized using Joule heating. Sci. Rep. 6, 28499 (2016).

6. Kanda, Y. A graphical representation of the piezoresistance coefficients in silicon. IEEE Trans. Electron Devices 29, 64-70 (1982).

7. Phan, H.-P. et al. Fundamental piezoresistive coefficients of p-type single crystalline 3C-SiC. Appl. Phys. Lett. 104, 111905 (2014).

8. Phan, H.-P. et al. The effect of strain on the electrical conductance of $\mathrm{p}$ type nanocrystalline silicon carbide thin films. J. Mater. Chem. C 3, 1172-1176 (2015).

9. Qamar, A. et al. A large pseudo-Hall effect in n-type 3C-SiC $\left(\begin{array}{lll}1 & 0 & 0\end{array}\right)$ and its dependence on crystallographic orientation for stress sensing applications. Mater. Lett. 213, 11-14 (2018).

10. Nguyen, T.-K. et al. Experimental investigation of piezoresistive effect in ptype 4HâĂŞSiC. IEEE Electron Device Lett. 38, 955-958 (2017).

11. Rowe, A., Donoso-Barrera, A., Renner, C. \& Arscott, S. Giant roomtemperature piezoresistance in a metal-silicon hybrid structure. Phys. Rev. Lett. 100, 145501 (2008).

12. Koumela, A. et al. Piezoresistance of top-down suspended Si nanowires. Nanotechnology 22, 395701 (2011).

13. He, R. \& Yang, P. Giant piezoresistance effect in silicon nanowires. Nat. Nanotechnol. 1, 42 (2006).

14. Md Foisal, A. R. et al. Pushing the limits of piezoresistive effect by optomechanical coupling in $3 \mathrm{C}-\mathrm{SiC} / \mathrm{Si}$ heterostructure. ACS Appl. Mater. Interfaces 9, 39921-39925 (2017).

15. Phan, H.-P. et al. Nano strain-amplifier: making ultra-sensitive piezoresistance in nanowires possible without the need of quantum and surface charge effects. Appl. Phys. Lett. 109, 123502 (2016)

16. Rowe, A. C. Silicon nanowires feel the pinch. Nat. Nanotechnol. 3, 311 (2008).

17. Milne, J., Rowe, A., Arscott, S. \& Renner, C. Giant piezoresistance effects in silicon nanowires and microwires. Phys. Rev. Lett. 105, 226802 (2010).

18. Zhou, J. et al. Flexible piezotronic strain sensor. Nano Lett. 8, 3035-3040 (2008).

19. Neuzil, P., Wong, C. C. \& Reboud, J. Electrically controlled giant piezoresistance in silicon nanowires. Nano Lett. 10, 1248-1252 (2010).

20. Yu, R., Wu, W., Ding, Y. \& Wang, Z. L. GaN nanobelt-based strain-gated piezotronic logic devices and computation. ACS Nano 7, 6403-6409 (2013).

21. Zhou, R., Hu, G., Yu, R., Pan, C. \& Wang, Z. L. Piezotronic effect enhanced detection of flammable/toxic gases by $\mathrm{ZnO}$ micro/nanowire sensors. Nano Energy 12, 588-596 (2015).

22. Pan, C., Yu, R., Niu, S., Zhu, G. \& Wang, Z. L. Piezotronic effect on the sensitivity and signal level of Schottky contacted proactive micro/nanowire nanosensors. Acs Nano 7, 1803-1810 (2013).

23. Wang, X. et al. Piezotronic effect modulated heterojunction electron gas in AlGaN/AlN/GaN heterostructure microwire. Adv. Mater. 28, 7234-7242 (2016). 
24. Zhang, K. \& Yang, Y. Thermo-phototronic effect enhanced InP/ZnO nanorod heterojunction solar cells for self-powered wearable electronics. Adv. Funct. Mater. 27, 1703331 (2017).

25. Wallmark, J. T. A new semiconductor photocell using lateral photoeffect. Proc. IRE 45, 474-483 (1957).

26. Dinh, T. et al. An on-chip SiC MEMS device with integrated heating, sensing, and microfluidic cooling systems. Adv. Mater. Interfaces 5, 1800764 (2018).

27. Sun, Y., Sun, G., Parthasarathy, S. \& Thompson, S. Physics of process induced uniaxially strained Si. Mater. Sci. Eng.: B 135, 179-183 (2006).

28. Qamar, A., Tanner, P., Dao, D. V., Phan, H.-P. \& Dinh, T. Electrical properties of p-type $3 \mathrm{C}-\mathrm{SiC} / \mathrm{Si}$ heterojunction diode under mechanical stress. IEEE Electron Device Lett. 35, 1293-1295 (2014).

\section{Acknowledgements}

The 3C-SiC material was developed and supplied by Leonie Hold and Alan Iacopi of the Queensland Microtechnology Facility, part of the Queensland node-Griffith-of the Australian National Fabrication Facility. A company established under the National Collaborative Research Infrastructure Strategy to provide nano and microfabrication facilities for Australia's researchers. The epitaxial SiC deposition was developed as part of Griffith Universities Joint Development Agreement with SPT Microtechnology, the manufacturer of the Epiflx production reactor. This work has been partially supported by Australian Research Council grants LP150100153 and LP160101553. T.D. is grateful for the support from Griffith University/Simon Fraser University Collaborative Travel Grant 2017 and Griffith University New Research Grant 2019.

\section{Author contributions}

T.N., T.D. and A.R.F. designed and carried out the experiments. T.D., H.-P.P. and T.-K.N. fabricated the samples. T.N. and T.D. analyzed the data. T.N., T.D., N.-T.N. and D.V.D. discussed the results. T.N., T.D. and D.V.D. co-wrote the paper. All authors commented on the manuscript.

\section{Additional information}

Supplementary Information accompanies this paper at https://doi.org/10.1038/s41467019-11965-5.

Competing interests: The authors declare no competing interests.

Reprints and permission information is available online at http://npg.nature.com/ reprintsandpermissions/

Peer review information Nature Communications thanks the anonymous reviewers for their contribution to the peer review of this work. Peer reviewer reports are available.

Publisher's note Springer Nature remains neutral with regard to jurisdictional claims in published maps and institutional affiliations.

\section{(c) (i)}

Open Access This article is licensed under a Creative Commons Attribution 4.0 International License, which permits use, sharing, adaptation, distribution and reproduction in any medium or format, as long as you give appropriate credit to the original author(s) and the source, provide a link to the Creative Commons license, and indicate if changes were made. The images or other third party material in this article are included in the article's Creative Commons license, unless indicated otherwise in a credit line to the material. If material is not included in the article's Creative Commons license and your intended use is not permitted by statutory regulation or exceeds the permitted use, you will need to obtain permission directly from the copyright holder. To view a copy of this license, visit http://creativecommons.org/ licenses/by/4.0/.

(C) The Author(s) 2019 